IJABBR- 2017- eISSN: 2322-4827

International Journal of Advanced Biological and Biomedical Research 5(2) (2017) 69-72

Journal homepage: www.ijabbr.com

\title{
N-Acetylcysteine Overdose After Acetaminophen Poisoning
}

\author{
Ali Akbar Razlansari ${ }^{1}$, Azadeh Jafrasteh ${ }^{* 2}$, Mahshid Garmsiri ${ }^{3}$ \\ ${ }^{1}$ Assistant Professor, Department of Pediatrics, Shiraz University of Medical Sciences, Shiraz, Iran \\ ${ }^{2}$ Department of Pediatrics, Lorestan University of Medical Sciences, Khorramabad, Iran \\ ${ }^{3}$ Medical Student, Student Research Committee, Lorestan University of Medical Sciences, Khorramabad, Iran
}

\section{ART I C L E IN F O}

\section{Article history:}

Received: 27 May 2017

Revised:21 Jun 2017

Accepted: 30 Jun 2017

ePublished: 30 Jul 2017

Key words:

$\mathrm{N}$-acetylcysteine

Overdose

Medication error

\section{A B S T R A C T}

$\mathrm{N}$-acetylcysteine (NAC) is a drug used widely and effectively in oral and intravenous forms as a specific antidote for acetaminophen poisoning. In children considering the toxic dose of acetaminophen ingested by the patient and the body weight, the physician prescribes a specific dose of NAC. There is always a risk of iatrogenic overdose of NAC which can cause different mild to severe temporary or permanent side effects, some of which can be life threatening. Here we report a rare case of iatrogenic NAC overdose following an error in calculation of the prescribed dose. The patient was a 2.5 years old boy that was brought to emergency room with overdose of acetaminophen syrup. The Physician prescribed 2 grams of intravenous NAC for him, but because of the miscalculation of the volume of the drug, the nurse infused about 20 grams of NAC (10 times the prescribed dose) to the patient. Immediately the patient became drowsy and developed decreased level of consciousness, stupor, repetitive intractable focal and generalized convulsions, decrease visual acuity and had prolonged hospitalization in intensive care unit and pediatric neurologic wards and perhaps will have permanent subtle intellectual and neurologic sequela. Considering the possible errors in the prescription and administration of some critical and less commonly used drugs especially those which have several different formulary and also considering their possible serious and life threatening side effects, it is recommended that the dose and volume of such medications be calculated meticulously by at least two trained persons. Also it is important to carry out the administration phases and check the patient's symptoms more attentively and cautiously.

\section{Introduction}

Acetaminophen is prevalently used to relieve pain and fever in children and adults and if it is prescribed and used based on standard doses of treatment protocols, there will be no risk for patients (Yang et al., 2015). Acetaminophen poisoning that is one of the most prevalent causes of poisoning in developed countries can be the result of prescription or overdose of this medicine. Acetaminophen poisoning is associated with different clinical manifestations (Mahmudi et al., 2013). The most important antidote to cure Acetaminophen poisoning is $\mathrm{N}$-Acetylcystein which has decreased the mortality rate due to Acetaminophen poisoning considerably. A similar diet therapy has been prescribed for all patients who suffer from Acetaminophen poisoning since 1970. This diet is regulated based on patients' weight and is accomplished in three phases: 1) the antidote is mixed in dextrose 5\%; then $150 \mathrm{ml}$ antidote is injected intravenously in the first 15 minutes per kilogram of

*Corresponding Author: Azadeh Jafrasteh, Department of Pediatrics, Lorestan University of Medical Sciences, Khorramabad, Iran (Azadeh_j2011@gmail.com) 
body weight. 2) $50 \mathrm{ml}$ antidote is injected to the patient in four hours per kilogram of body weight. 3) Finally, 100 $\mathrm{ml}$ antidote is injected in 16 hours per kilogram of body weight (Prescott et al., 1977; Ferner et al., 2011; Heard, 2008; Bateman et al., 2014).

In some cases, there were side effects of this antidote which occurred mostly in the first 15 minutes after injection. They included vomiting and anaphylactic reactions (Whyte et al., 2007; Lynch et al., 2004; Waring et al., 2006; Pakravan et al., 2008; Schmidt, 2013). On the other hand, Acetaminophen poisoning can per se be led to nausea and vomiting that intensity of these side effects becomes higher if antidote is used (Jalali et al., 2000). In its therapeutic dose, NAC is safe for the patient but danger of side effects will be increased in case of inappropriate prescription. In this case report, we present a rare case of iatrogenic NAC overdose following an error in calculation of the prescribed dose in a 2.5 year-old child.

\section{Case report}

The patient was a 2.5 years old boy that was brought to emergency room by his parents with overdose of acetaminophen syrup. The Physician prescribed 2 grams of intravenous NAC for him, but because of the miscalculation of the volume of the drug, the nurse infused about 20 grams of NAC (10 times the prescribed dose) to the patient. Immediately the patient became drowsy and developed decreased level of consciousness, stupor, repetitive intractable focal and generalized convulsions, decrease visual acuity and had prolonged hospitalization in intensive care unit and pediatric neurologic wards and perhaps will have permanent subtle intellectual and neurologic sequela. Despite this problem, vital signs of the patient were not changed perceptibly. The parents mentioned that the patient had no history of mental disease and hospitalization. The parents had no family relationship and had an average socio-economic status. The patient was transferred to the Intensive Care Unit (ICU) sector given these symptoms. After transferring to ICU, NAC overdose was diagnosed; thus, injection of the medicine was stopped quickly and supportive therapy was started. The symptoms were eliminated after three days and the patient was finally discharged with parental consent. Post-treatments after the patient's discharge indicated his/her full health.

\section{Discussion}

Therapeutic and safe dose of Acetaminophen in adults is maximally $60 \mathrm{ml} / \mathrm{kg}$ and in children is between 20 to 50 $\mathrm{ml} / \mathrm{kg}$. Using doses more than the above ones will be led to Acetaminophen poisoning. This has been one of the most prevalent causes of self-poisoning in the world. Its annual outbreak in the United States is 30000 persons. Acetaminophen poisoning in the most critical case can be led to Fulminant hepatic failure and the patient's death but mortality rate and appearance of side effects have been considerably decreased due to the existence of NAC as an effective antidote (Diallo et al., 2016; Manthripragada et al., 2011, Green et al., 2013; Bateman et al., 2014; Mowry et al., 2014; Duffull et al., 2013; Buckley et al., 2015; Prescott et al., 1971). This antidote is available in both oral and injectable form. Its oral form is absorbed during half an hour with maximum degree of absorption through the digestive system but its injectable form is preferable because of gastrointestinal side effects and sometimes intensification of poisoning symptoms. Nowadays, it is routinely used in Acetaminophen poisoning. Studies in the last decade show an extensive range of side effects because of using NAC to cure Acetaminophen poisoning (side effects such as gastrointestinal side effects, tachycardia, skin rash, bronchospasm, hypotension, and systematic anaphylactic reactions). For instance, prospective studies in the recent decade have revealed that side effects of this medicine is between 29 and 77 percent and degree of systemic hypersensitivity reactions due to its prescription is between 14 and 75 percent (Jalali et al., 2000; Isbistera et al., 2015; Wright et al., 1996; Heard et al., 2011; Kante et al., 2006; Brok et al., 2006). In the above patient, side effects such as decreased level of consciousness, reduction of visual acuity as well as repeated epileptic seizures were observed. Of course, it is notable that these side effects cannot be just considered due to side effects of this medicine; rather, high dose of the medicine can be a more suitable reason. In another case report in which a patient with Glucose 6-phosphate dehydrogenase enzyme deficiency (G6PD) and over-injection of NAC was introduced, it was stated that overprescription of this antidote has been led to hemolysis, thrombocytopenia, kidney failure, and finally death of the patient (Mahmoudi et al., 2015).

The medicine use was stopped as soon as symptoms were appeared in the above patient and then supportive therapy began. In a prospective study by Isbistera et al, it has been argued that side effects of this medicine will be observed in 48 percent of cases if the patient is toxic who suffers from NAC poisoning but if injection of the medicine is stopped before full toxicity, side effects will be occurred in 28 percent of cases (Isbistera et al., 2015). In the above patient, injection of the medicine was stopped before the patient's toxicity and immediately after appearance of the symptoms. After 3 days, clinical symptoms of the patient were interrupted and he/she was discharged with parental consent. As it was mentioned previously, post-treatments after the patient's discharge indicated his/her full health.

The main issue that should be considered in this paper is occurrence of medical error which could be led to the patient's death. According to the statistics, annual mortality rate due to medical error is 440000 cases and rate of harms to patients is one million cases. In addition to injuries statistics, results of various studies in the US demonstrate that the annual financial load which is imposed to the health system of this country due to medical error is between 17 and 29 billion dollars 
(Slonim et al., 2003; Zaboli et al., 2015). On the other side, previous studies show that 3 to 17 percent of inpatients will suffer from side effects which are related to medical errors (Zaboli et al., 2015). In explaining the causes of medical errors, studies done by Cramer et al. and Balas et al. can be referred. In their study in Germany, Cramer et al. argued that high workload, high business hours and lack of health service personnel are the most important causes for the occurrence of medical errors (Cramer et al., 2013). Likewise, Balas et al. believed that weariness of nurses is the most important factor in occurrence of medical error (Balas et al., 2004). In a study that was carried out by Wakefield et al, it was stated that nurses may not report the medical error in some cases because of fear of punishment by authorities and this will harm the patient (Wakefield et al., 1999). Different studies show that degree of error in all sectors of a hospital is not the same. For instance, the final results in a study indicated that 48 percent of medical errors are occurred in the emergency department. This result can show the effect of weariness and high workload of personnel in this sector (Kalisch et al., 2009). In the above patient, we observed occurrence of a medical error by a nurse in the emergency department. But the error was reported timely and an appropriate treatment was adopted to improve the patient's status.

The important point is that medical error is the inseparable part of health system for which managerial and preventive techniques should be adopted. For instance, hiring more employees with fewer business hours in sectors which have the highest statistics of medical error as well as providing conditions for the physician to supervise how treatment actions are accomplished by the nurse can be effective in this regard.

\section{References}

Yang, X., Salminen, W., Shi, Q., Greenhaw, J., Gill, P., Bhattacharyya, S., et al. 2015. Toxicology and Applied Pharmacology. 284, 180-187.

Mahmudi, Gh., Anbari, Kh., Obeidavi, Z., 2013. Prevalence of clinical manifestations of poisoning in children admitted to hospitals of Khorramabad in 2011. Iranian Journal of Toxicology, 7 (22).

Prescott, L.F., Illingworth, R.N., Critchley, J.A., Stewart, M.J., Adam, R.D., Proudfoot, A.T., 1977. Treatment of paracetamol (acetaminophen) poisoning with $\mathrm{N}$ acetylcysteine. Lancet, 2, 432-434.

Ferner, R.E., Dear, J.W., Bateman, D.N., 2011. Management of paracetamol poisoning. BMJ, 342, d2218.

Heard, K.J., 2008. Acetylcysteine for acetaminophen poisoning. N. Engl. J. Med., 359, 285-292.

Bateman, D.N., Carroll, R., Pettie, J., Yamamoto, T., Elamin, M., Peart, L., Dow, M., Coyle, J., Cranfield, KR., Hook, C.,
Sandilands, EA., Veiraiah, A., Webb, D., Gray, A., Dargan, PI., Wood, DM., Thomas, SH., Dear, JW., Eddleston, M., 2014. Effect of the UK's revised paracetamol poisoning management guidelines on admissions, adverse reactions and costs of treatment. Br. J. Clin. Pharmacol., 78(3), 610618.

Whyte, I.M., Francis, B., Dawson, A.H., 2007. Safety and efficacy of intravenous $\mathrm{N}$-acetylcysteine for acetaminophen overdose: analysis of the Hunter Area Toxicology Service (HATS) database. Curr. Med. Res. Opin., 23, 2359-68.

Lynch, R.M., Robertson, R., 2004. Anaphylactoid reactions to intravenous $\mathrm{N}$-acetylcysteine: a prospective case controlled study. Accid. Emerg. Nurs., 12, 10-5.

Waring, W.S., Pettie, J.M., Dow, M.A., Bateman, D.N., 2006. Paracetamol appears to protect against $\mathrm{N}$-acetylcysteineinduced anaphylactoid reactions. Clin. Toxicol., 44, 441-2.

Pakravan, N., Waring, W.S., Bateman, D.N., 2008. Risk factors and mechanisms of anaphylactoid reactions to acetylcysteine in acetaminophen overdose. Clin. Toxicol., $46,697-702$.

Schmidt, L., 2013. Identification of patients at risk of anaphylactoid reactions to $\mathrm{N}$ - Acetylcysteine in the treatment of paracetamol overdos. Clin. Toxicol., 51, 46772.

Jalali, N., Kariman, N., Snaei zadeh, H., 2000. Evaluation of NAC treatment side effect in acetaminophen poisoning. Journal of Shahid Sadoughi University of Medical Sciences, 9(3), 25-28.

Diallo, A., Eliassou, M., Djadou, K., Potchoo, Y., Creppy, E.E., 2016. Accidental Acute Poisoning of two Children by Paracetamol-Codeine $(1000 \mathrm{Mg} / 60 \mathrm{Mg})$ Association - A Case Report. Open J. Clin. Toxicol., 1(1), 001-003.

Manthripragada, A.D., Zhou, E.H., Budnitz, D.S., Lovegrove, M.C., Willy, M.E., 2011. Characterization of acetaminophen overdose-related emergency department visits and hospitalizations in the United States. Pharmacoepidemiol. Drug. Saf., 20(8), 819-826.

Green, J., Heard, K., Reynolds, K., Albert, D., 2013. Oral and Intravenous Acetylcysteine for Treatment of Acetaminophen Toxicity: A Systematic Review and Metaanalysis. Western Journal of Emergency Medicine, 14(3), 218-226.

Bateman, D.N., Dear, J.W., Thanacoody, H.K., Thomas, H.L., Eddleston, M., Sandilands, E.A., Coyle, J., Cooper, J.G., Rodriguez, A., Butcher, I., Lewis, S.C., Vliegenthart, A.D., Veiraiah, A., Webb, D.J., Gray, A., 2014. Reduction of adverse effects from intravenous acetylcysteine 
treatment for paracetamol poisoning: a randomized controlled trial. Lancet, 383, 697-704.

Mowry, J.B., Spyker, D.A., Cantilena, L.R Jr., McMillan, N., Ford, M., 2014. 2013 Annual Report of the American Association of Poison Control Centers' National poison data system (NPDS): 31st annual report. Clin. Toxicol. (Phila)., 52: 1032-1283.

Duffull, S.B., Isbister, G.K., 2013. Predicting the requirement for $\mathrm{N}$-acetylcysteine in paracetamol poisoning from reported dose. Clin. Toxicol. (Phila)., 51, 772-776.

Buckley, N.A., Whyte, I.M., Dawson, A.H., 2015. A prospective cohort study of trends in self-poisoning, Newcastle, Australia, 1987-2012: plus ca change, plus c'est la meme chose. Med. J. Aust., 202, 438-442.

Prescott, L.F., Roscoe, P., Wright, N., Brown, S.S., 1971. Plasma-paracetamol half-life and hepatic necrosis in patients with paracetamol overdosage. Lancet, 1(7698), 519-22.

Isbistera, G., Downesa, M., Mcnamarab, K., Berlinga, I., Whytea, I., Pagea, C., 2015. A prospective observational study of a novel 2-phase infusion protocol for the administration of acetylcysteine in paracetamol poisoning. Clinical toxicology, 54(2), 120-126.

Wright, R.O., Perry, H.E., Woolf, A.D., Shannon, M.W., 1996. Hemolysis after acetaminophen overdose in a patient with glucose-6-phosphate dehydrogenase deficiency. J. Toxicol. Clin. Toxicol., 34, 731-734.

Heard, K., Schaeffer, T.H., 2011. Massive acetylcysteine overdose associated with cerebral edema and seizures. Clin. Toxicol., 49, 423-425.
Kante, M.Z., 2006. Comparison of oral and IV acetylcysteine in the treatment of acetaminophen poisoning. Am. J. Health Syst. Pharm., 63, 1821-1827.

Brok, J., Buckley, N., Gluud, C., 2006. Interventions for paracetamol (acetaminophen) overdose. Cochrane Database Syst. Rev., 2, CD003328.

Mahmoudi, Gh., Astaraki, P., Zafar Mohtashami, A., Ahadi, M., 2015. N-acetylcysteine overdose after acetaminophen poisoning. International Medical Case Reports Journal, 8, 65-69.

Slonim, A., LaFleur, B., Ahmed, W., Joseph, J., 2003. Hospital-Reported Medical Errors in Children. Pediatrics J., 111 (3), 617-621.

Zaboli, R., Abbaszadeh, A., Shahabinejad, M., 2015. Assessing the barriers of error reporting from perspective of nurses in Kerman hospitals. Medical Ethics Journal, 34 (9), 31-53.

Cramer, H., Pohlabeln, H., Habermann, M., 2013. Factors causing or influencing nursing errors as perceived by nurses: findings of a cross- sectional study in German nursing homes and hospitals. Journal of Public Health, 21(2):145-153.

Balas, M.G., Scott, L.D., Rogers, A.E., 2004. The prevalence and nature of errors and near errors reported by hospital staff nurses. Appl. Nurs. RES., 17(4), 224-33.

Wakefield, D.S., Wakefield, B.J., Uden-Holman, T., Borders, T., Blegen, M., Vaughn, T., 1999. Understanding why medication administration errors may not be reported. Am. J. Med. Qual., 14(2), 81-88.

Kalisch, B.J., Landstrom, G., Williams, R.A., 2009. Missed nursing care: errors of omission. Nursing Outlook, 57(1), 3-9.

How to cite this manuscript: Ali Akbar Razlansari, Azadeh Jafrasteh, Mahshid Garmsiri. N-Acetylcysteine Overdose After Acetaminophen Poisoning. International Journal of Advanced Biological and Biomedical Research 5(2), 2017, 69-72. D0I: 10.18869/IJABBR.2017.455. 